\title{
Effect of uncontrolled hyperglycemia on levels of adhesion molecules in patients with diabetes mellitus type $2^{*}$
}

\author{
Barbara RUSZKOWSKA-CIASTEK ${ }^{\dagger 1}$, Alina SOKUP ${ }^{1}$, Tomasz WERNIK ${ }^{1}$, Zofia RUPRECHT ${ }^{2}$, \\ Barbara GÓRALCZYK ${ }^{1}$, Krzysztof GÓRALCZYK ${ }^{1}$, Grażyna GADOMSKA ${ }^{1}$, Danuta ROŚĆ ${ }^{1}$ \\ $\left({ }^{1}\right.$ Department of Pathophysiology, Faculty of Pharmacy, Nicolaus Copernicus University in Torun', \\ Collegium Medicum in Bydgoszcz, Bydgoszcz PL 85-094, Poland) \\ $\left({ }^{2}\right.$ Department of Endocrinology and Diabetology, Faculty of Medicine, Nicolaus Copernicus University in Toruń, \\ Collegium Medicum in Bydgoszcz, Bydgoszcz PL 85-094, Poland) \\ ${ }^{\dagger}$ E-mail: ruszkowska.basia@gmail.com
}

Received Aug. 7, 2014; Revision accepted Nov. 13, 2014; Crosschecked Apr. 9, 2015

\begin{abstract}
Objective: Uncontrolled diabetes has become a major cause of mortality and morbidity by reason of vascular angiopathy. The aim of this study was to evaluate the concentrations of soluble forms of vascular adhesion molecule-1 (sVCAM-1), intercellular adhesion molecule-1 (sICAM-1), E-selectin, and thrombomodulin in patients with well-controlled and uncontrolled diabetes type 2. Methods: The study was conducted on 62 patients with diabetes. Group I consisted of 35 patients with well-controlled diabetes. The second group included 27 patients with uncontrolled diabetes with micro-albuminuria. A control group was made up of 25 healthy volunteers. The concentrations of sVCAM-1, sICAM-1, sE-selectin, and soluble thrombomodulin were assayed in plasma. Serum concentration of creatinine was measured and the plasma concentrations of fasting glucose and glycated hemoglobin (HbA1c) determined. Results: Lower concentrations of ICAM-1 were found in the group of uncontrolled diabetes patients compared with those with well-controlled disease. In patients with uncontrolled diabetes, VCAM-1 levels were significantly higher compared with the group with well-controlled diabetes. In patients with uncontrolled diabetes a positive correlation was obtained between glomerular filtration rate and sE-selectin and a negative correlation between the levels of creatinine and ICAM-1, although there was a positive correlation between (HbA1c) and ICAM-1. Conclusions: The study confirmed the participation of the inflammatory process associated with impaired vascular endothelial function in the pathogenesis of type 2 diabetes. The opposite effect of uncontrolled hyperglycemia on adhesion molecules suggests different functions of VCAM-1 and ICAM-1 in complications of diabetes.
\end{abstract}

Key words: Diabetic kidney disease, Glomerular filtration rate, Hyperfiltration doi: $10.1631 /$ jzus.B1400218

Document code: A

CLC number: R587.1

\section{Introduction}

A properly functioning endothelium inhibits the adhesion of platelets and leukocytes to the surface of the vessel wall while maintaining a balance between pro-inflammatory, anti-inflammatory, pro-coagulant and anti-coagulant activity. Endothelial cells also

\footnotetext{
" Project supported by the Nicolaus Copernicus University, Collegium Medicum in Bydgoszcz, Poland

DDCID: Barbara RUSZKOWSKA-CIASTEK, http://orcid.org/00000003-4245-2403; Danuta ROŚĆ, http://orcid.org/0000-0002-2971-9882 (C) Zhejiang University and Springer-Verlag Berlin Heidelberg 2015
}

produce specific adhesion molecules, which play an important role in the regulation of inflammation and which include vascular adhesion molecule-1 (VCAM-1), intercellular adhesion molecule-1 (ICAM-1), and leukocyte adhesion molecule (Hadi and Suwaidi, 2007). However, one of the most sensitive and specific markers of endothelial damage is thrombomodulin (TM), an integral transmembrane glycoprotein. $\mathrm{TM}$ is an important protein with vasoprotective properties acting as an inhibitor of thrombin, which loses prothrombotic properties when bound to TM (Głowińska-Olszewska et al., 2007). 
Vascular endothelial damage is a major cause of vascular complications in diabetes (Hadi and Suwaidi, 2007). Hyperglycemia is an important factor, which leads to vascular changes. In the state of hyperglycemia, glucose molecules are non-enzymatically coupled with the lateral chains of lysine in proteins (the process of protein glycation). Glycated proteins have a strong affinity to immunoglobulins or complement components activating the process of inflammation, which damages the vessel wall (Szmit and Opolski, 2006; Hadi and Suwaidi, 2007). Another phenomenon, which plays an important role in vascular remodeling, is oxidative stress such as the overproduction of reactive oxygen species stimulated by lipid abnormalities, pro-inflammatory cytokines, and the activation of the renin angiotensin system (Żukowska-Szczechowska and Wystrychowski, 2007).

Diabetic kidney disease is a complication of diabetes, both types 1 and 2, which may develop in $20 \%-30 \%$ of patients with diabetes. The number of patients treated with renal replacement therapy due to diabetic nephropathy has increased significantly in recent years. This includes patients with nephropathy, especially in the course of type 2 diabetes. Diabetic nephropathy is characterized by overt proteinuria in patients with type 1 or 2 diabetes with the absence of other kidney diseases. It is recognized when vascular, post-inflammatory changes, diffuse nodular or less frequently exudative glomerulosclerosis and hyalinizing alterations are observed in the blood vessels along with gradual reduction in glomerular filtration rate (GFR) (Szmit and Opolski, 2006; Głowińska and Małyszko, 2008).

In the course of diabetes changes occur in the concentration of several markers of endothelial damage, such as increased levels of von Willebrand factor, TM, sE-selectin, tissue plasminogen activator, and adhesion molecules ICAM-1 and VCAM-1. Increased concentration of ICAM-1 is associated with an increased risk of type 2 diabetes (Hadi and Suwaidi, 2007). Fluctuations in the concentration of adhesion molecules may be a useful indicator of the development of atherosclerotic plaques from the early phase in diabetic patients since E-selectin, VCAM-1, and ICAM-1 facilitate the adhesion of leukocytes to endothelium (Pupek-Musialik et al., 2005; Hadi and Suwaidi, 2007; Lu et al., 2011).

The aim of this study was to evaluate the con- centrations of selected inflammatory markers associated with vascular endothelium: soluble forms of VCAM-1 (sVCAM-1), ICAM-1 (sICAM-1), E-selectin (sE-selectin), and TM (sTM) in patients with wellcontrolled type 2 diabetes and uncontrolled diabetes with micro-albuminuria.

\section{Materials and methods}

The study was performed on 62 patients with type 2 diabetes under the care of the Endocrinology and Diabetology Clinic of the A. Jurasz University Hospital, Poland. The first group (I) consisted of 35 patients with well-controlled type 2 diabetes (17 females aged $47-75$ years (mean age 63 years) and 18 males aged 46-79 years (mean age 64 years)). The diabetic compensation criterion in group I consisted of glycated hemoglobin (HbAlc) $\leq 48 \mathrm{mmol} / \mathrm{mol}$ $(\leq 6.5 \%)$ and no vascular complications, assessed by determining the concentration of albumin in the urine (to exclude diabetic kidney disease), an eye examination (to exclude the presence of diabetic retinopathy), as well as no symptoms of ischemic heart disease, no symptoms of lower limb ischemia (measured by testing the pressure of the arteries of the lower limbs to calculate the ankle-brachial index: selected patients had an index of 0.90-1.15), and no diabetic foot syndrome. The second group (II) covered 27 patients with uncontrolled type 2 diabetes with micro-albuminuria (10 females aged 4672 years (mean age 62 years) and 17 males aged 48-74 years (mean age 63 years)). The qualification criterion of patients for the study was $\mathrm{HbAlc} \geq$ $59 \mathrm{mmol} / \mathrm{mol}(\geq 7.5 \%)$ and identifying considerable albuminuria in two of three measures after bacteriological urine examination and exclusion of inflammation or overt proteinuria. In these patients, GFR was $40-60 \mathrm{ml} / \mathrm{min}$ per $1.73 \mathrm{~m}^{2}$. GFR was calculated according to the modification of diet in the renal disease equation.

The medical history included the general conditions of the patients and the occurrence of co-existing diseases. All the patients received anti-hyperglycemic treatment: insulin, metformin, and sulfonylureaderivatives, depending on the indication, and all the patients have also received statins and angiotensinconverting-enzyme inhibitor drugs. 
The control group consisted of 25 healthy volunteers ( 12 females and 13 males, mean age 52 years).

Informed consent was obtained from all patients. The research was approved by the Bioethics Committee of Collegium Medicum in Bydgoszcz, the Nicolaus Copernicus University in Torun (No. KB 366/2006), Poland.

Venous blood $(4.5 \mathrm{ml})$ for tests of sVCAM-1, sICAM-1, sTM, and sE-selectin was collected in a fasting state into cooled tubes (Becton Dickinson Vacutainer $^{\circledR}$ System, Plymouth, UK) containing $0.13 \mathrm{~mol} / \mathrm{L}$ trisodium citrate after a 30 -min rest between 7:30 a.m. and 9:30 a.m. and after a 12-h overnight fast. The blood samples were immediately mixed and centrifuged at $3000 \mathrm{~g}$ at $4{ }^{\circ} \mathrm{C}$ for $20 \mathrm{~min}$. The platelet-poor plasma was divided into $200 \mu \mathrm{l}$ Eppendorf-type tubes and the samples were then frozen at $-86{ }^{\circ} \mathrm{C}$ (according to the manufacturer's procedures) until assayed within six months.

To determine concentration of creatinine, blood was collected in a $4.5-\mathrm{ml}$ tube without anticoagulant. It was centrifuged at $3000 \mathrm{~g}$ for $20 \mathrm{~min}$ at $4{ }^{\circ} \mathrm{C}$ and subjected to further analytical procedure. To measure fasting glucose, blood was collected in a $4.5-\mathrm{ml}$ tube with sodium fluoride ethylenediaminetetraacetic acid (EDTA). The plasma was centrifuged at $2000 \mathrm{~g}$ for $10 \mathrm{~min}$ at $4{ }^{\circ} \mathrm{C}$ and subjected to further analytical procedure. In addition, $4.5 \mathrm{ml}$ of blood was collected into tubes with sodium EDTA in order to determine the level of HbA1c, and obtained plasma was directly subjected to further analytical procedure.

The concentrations of sVCAM- 1 and SICAM-1 were assayed using the test (Human SVCAM-1 and Human SICAM-1, respectively) provided by Bender MedSystems GmbH (Vienna, Austria). The sTM concentration was measured with the test provided by sTM IMUBIND $^{\circledR}$ Thrombomodulin (American Diagnostica Inc., Stamford, USA), and sE-selectin concentrations using the test Human sE-selectin provided by Bender MedSystems GmbH (Vienna, Austria). The principle of all the methods was based on enzyme-linked immune sorbent assay (ELISA).
The parameters of fasting glucose, creatinine, and $\mathrm{HbA} 1 \mathrm{c}$ test were determined using the Abbott Clinical Chemistry Analyzer ${ }^{\circledR}$ Architect c8000. Enzymatic and immunoturbidimetric methods were used to measure the concentrations of fasting glucose, creatinine, and $\mathrm{HbAlc}$, respectively.

The statistical analysis was performed with Statistica 10.0 software (StatSoft ${ }^{\circledR}$, Cracow, Poland). The Shapiro-Wilk test was used to assess the normality of the distribution. The variables show a different distribution from normal, and thus the median, lower quartile (Q1), and upper quartile (Q3) were used. To identify the significance of the differences between the groups, analysis of variance (ANOVA) Kruskal-Wallis post hoc test was used. To assess the correlation between the parameters, the Pearson correlation coefficient was used. The $P$-values of $<0.05$ were considered significant.

\section{Results}

Clinical specific features of the patients with well-controlled type 2 diabetes and uncontrolled diabetes are showed in Table 1.

Table 2 shows the markers of inflammation and endothelial damage analyzed in the patients with well-controlled type 2 diabetes (Group I), patients with uncontrolled diabetes with micro-albuminuria (Group II), and the control group (Group III). In the patients with type 2 diabetes, higher levels of sICAM-1 were recorded than in the control group; the differences were significant (Group I, $P=0.000002$; Group II, $P=0.0021)$. Significantly higher concentrations of sICAM-1 were found in Group I, compared with Group II $(P=0.0118)$. In both groups of patients with diabetes, sVCAM-1 levels were higher, compared with the control group (Group I, $P=0.0281$; Group II, $P=0.0001)$. However, in the patients of Group I, sVCAM-1 levels were significantly lower compared with Group II $(P=0.0375)$. The concentrations of sE-selectin were higher in both groups of diabetic

Table 1 Selected clinical data of patients with well-controlled type 2 diabetes and uncontrolled diabetes

\begin{tabular}{cccccccc}
\hline Group & $\begin{array}{c}\text { Mean age } \\
(\text { year })\end{array}$ & $\begin{array}{c}\text { Arterial } \\
\text { hypertension }\end{array}$ & Hyperlipidemia & $\begin{array}{c}\text { Mean duration of } \\
\text { disease (year) }\end{array}$ & $\begin{array}{c}\text { HbA1c IFCC } \\
(\mathrm{mmol} / \mathrm{mol})\end{array}$ & $\begin{array}{c}\text { HbA1c NGSP } \\
(\%)\end{array}$ & $\begin{array}{c}\text { GFR } \\
(\mathrm{ml} / \mathrm{min})\end{array}$ \\
\hline I $(n=35)$ & 64 & 35 & 25 & 8.1 & 48 & 6.5 & 82 \\
II $(n=27)$ & 63 & 27 & 27 & 12.6 & 67 & 8.3 & 54 \\
III $(n=25)$ & 52 & & & & $<26$ & $<4.5$ & $80-120$ \\
\hline
\end{tabular}

IFCC: International Federation of Clinical Chemistry; NGSP: National Glycohemoglobin Standardization Program 
Table 2 Concentrations of sVCAM-1, sICAM-1, sTM, and sE-selectin in the patients with well-controlled type 2 diabetes and uncontrolled diabetes, compared with the controls

\begin{tabular}{ccccc}
\hline Group & sICAM-1 $(\mathrm{ng} / \mathrm{ml})$ & sVCAM-1 $(\mathrm{ng} / \mathrm{ml})$ & sE-selectin $(\mathrm{ng} / \mathrm{ml})$ & sTM $(\mathrm{ng} / \mathrm{ml})$ \\
\hline I $(n=35)$ & 372.10 & 709.45 & 30.90 & 2.40 \\
& $275.10 / 503.70$ & $540.60 / 1081.10$ & $21.20 / 38.80$ & $1.84 / 2.88$ \\
II $(n=27)$ & 288.60 & 1152.20 & 57.42 & 3.36 \\
& $223.90 / 341.80$ & $753.40 / 1569.90$ & $42.80 / 76.60$ & $2.80 / 3.90$ \\
III $(n=25)$ & 201.82 & 221.70 & 21.10 & 2.50 \\
& $161.46 / 244.79$ & $110.30 / 753.40$ & $9.90 / 24.59$ & $1.78 / 4.60$ \\
\hline$P$-value & & & 0.00001 & 0.0004 \\
I vs. II & 0.0118 & 0.0375 & 0.0029 & 0.1925 \\
I vs. III & 0.000002 & 0.0281 & 0.000001 & 0.3374 \\
II vs. III & 0.0021 & 0.0001 & & \\
\hline
\end{tabular}

The values of the parameters are shown as median (Me) and lower (Q1)/upper (Q3) quartile

Table 3 Correlations between analyzed parameters with GRF, creatinine and HbA1c levels in patients with uncontrolled diabetes type 2 with micro-albuminuria

\begin{tabular}{|c|c|c|c|c|c|c|c|c|}
\hline \multirow{2}{*}{ Parameter } & \multicolumn{2}{|c|}{ sICAM-1 } & \multicolumn{2}{|c|}{ sVCAM-1 } & \multicolumn{2}{|c|}{ sE-selectin } & \multicolumn{2}{|c|}{ sTM } \\
\hline & $r$ & $P$ & $r$ & $P$ & $r$ & $P$ & $r$ & $P$ \\
\hline GFR & 0.098 & 0.708 & -0.7484 & 0.146 & 0.6771 & $0.008^{*}$ & -0.4594 & 0.064 \\
\hline Creatinine & -0.4728 & $0.048^{*}$ & 0.5644 & 0.243 & 0.2681 & 0.334 & 0.1021 & 0.687 \\
\hline $\mathrm{HbAlc}$ & 0.4325 & $0.044^{*}$ & -0.6913 & 0.058 & -0.0007 & 0.998 & -0.1245 & 0.581 \\
\hline
\end{tabular}

* Significant correlation at $P<0.05$

patients, compared with the control group; the differences were significant (Group I, $P=0.0029$; Group II, $P=0.000001)$. In the patients of Group II, the concentrations of sE-selectin and sTM were significantly higher than in the diabetic patients of Group I ( $P=0.00001$ and $P=0.0004$, respectively).

Table 3 shows the correlations between selected parameters. We have found a significant positive correlation between the concentration of sE-selectin and GFR in patients of Group II ( $P=0.008)$, negative correlation between the levels of creatinine and sICAM-1 ( $P=0.048)$, but positive correlation between HbAlc and sICAM-1 $(P=0.044)$.

\section{Discussion}

The dysfunction of vascular endothelium plays an important role in the potential pathogenesis of the chronic microvascular complications in the kidneys in patients with or without diabetes mellitus with simultaneous micro-albuminuria. Endothelial dysfunction markers, such as the SVCAM-1, sICAM-1, von Willebrand factor, and microvascular hyper-reactivity, are observed in patients with type 2 diabetes even before the occurrence of micro-albuminuria (Karalliedde and Gnudi, 2011). The increase in the concentrations of sVCAM-1 and sICAM-1 reflects the degree of endothelial damage and the progression of diabetic nephropathy in the course of diabetes type 1 and 2 (Empen et al., 2003; Lu et al., 2011).

The present study showed that in type 2 diabetes, there occur higher levels of sICAM-1, sE-selectin, and sVCAM-1. In the patients of Group II noted higher levels of sVCAM-1, sE-selectin, and sTM but lower sICAM-1 concentration compared with the patients of Group I.

In the study reported by Pupek-Musialik et al. (2005) a group of 38 obese patients with metabolic syndrome and hyperglycemia had significantly higher levels of ICAM-1, compared with a control group. Also, Bruno et al. (2008) studying a group of 49 patients with type 2 diabetes (21 with normoalbuminuria, 28 with micro-albuminuria) reported that the concentrations of SICAM- 1 and SVCAM-1 were higher than in the control group. Guler et al. (2002) studying 93 patients with type 2 diabetes (50 with diabetic nephropathy and 43 without nephropathy) found higher mean levels of sICAM-1 in the patients with diabetic nephropathy, compared with the patients without nephropathy. Lu et al. (2011), investigating a group of 72 patients with type 2 diabetes, including 30 with diabetic nephropathy, obtained significantly higher levels of sICAM-1 in 
the patients with diabetic nephropathy, compared with the control group. However, no differences were observed in comparison with the group of the patients without nephropathy. According to Luis-Rodriguez et al. (2012), renal expression of ICAM-1 increased in the course of diabetic nephropathy and is associated with the progression of renal damage. They claim that this can suggest that modulation of the level of ICAM-1 by reducing its expression or blocking the receptor activating it can be related to the effects of the therapy in diabetic nephropathy.

However, Mizia-Stec et al. (2003) did not observe any significant differences in the concentrations of adhesion molecules, ICAM-1 and VCAM-1, in the patients with diabetes, compared with controls. Nevertheless, Stehouwera et al. (2002) showed higher level of VCAM-1 in patients with type 2 diabetes accompanied by overt micro-albuminuria. Similar results were reported in the patients with diabetic nephropathy by Marcos et al. (2001). However, antioxidant therapy with vitamin $\mathrm{C}$ and $\mathrm{E}$ led to a substantial reduction in its concentration in the blood of patients with diabetes. Significantly higher concentrations of sVCAM-1 in patients with diabetic nephropathy, compared with the control group and the group of diabetic patients without nephropathy, were noted by Lu et al. (2011).

The ambiguous results regarding, in particular, the concentrations of sVCAM- 1 and sICAM- 1 in patients with type 2 diabetes mellitus with microalbuminuria and without associated vascular complications are difficult to explain on the basis of the present results, yet one may assume that they can be due to the treatment of patients with insulin and other drugs. However, on the basis of data from the literature, it can be concluded that the level of adhesion molecules increases in obstructed kidneys at a different time. ICAM expression increases very rapidly at about the 3rd hour and remains high at around $90 \mathrm{~d}$ after obstruction, while the level of VCAM increases 2-3 d after obstruction (Grande et al., 2010).

The resulting positive correlation between GFR and the level of sE-selectin can be an excellent progression indicator for patients with micro-albuminuria since high levels of sE-selectin, as a marker of activated inflammatory process and hyperfiltration (increase of GFR), will lead to the simultaneous deterioration of kidney function and in consequence to diabetic nephropathy. Moreover, in this study, it is interesting that there is a positive correlation between the levels of HbAlc and sICAM-1 but a negative correlation between the levels of creatinine and sICAM-1. On this basis, it can be assumed that there is a level of pro-inflammatory molecules which are essential for proper control of glycemia.

In diabetes types 1 and 2 in response to hyperglycemia, the activity of $\mathrm{Ca}^{2+}$-dependent calpainprotein increases. The inhibition of calpain activity decreases the expression of pro-inflammatory adhesion molecules, ICAM and VCAM, on the endothelial surface during hyperglycemia (Stalker et al., 2003). In addition, the inhibition of protein kinase reduces the interaction between leukocytes and the vascular endothelium through the suppression of endothelial cell surface expression in response to oxidative stress (Booth et al., 2002). Perhaps those key reactions, which result in inhibition, can lead to reduction of the inflammatory process in the kidney glomerulus.

E-selectin is considered a factor indicating the activation of vascular endothelial cells; its expression is observed on inflamed endothelial cells in response to pro-inflammatory cytokines (Empen et al., 2003). In the present study, higher levels of sE-selectin were recorded in diabetic patients compared with controls. Similarly, Mizia-Stec et al. (2003) noted significantly higher levels of sE-selectin in the patients with type 2 diabetes, compared with controls. In the case-cohort study by the Monitoring of Trends and Determinants in Cardiovascular Disease (MONICA)/Cooperative research, men and women with elevated levels of sE-selectin demonstrated a significantly higher risk of type 2 diabetes after multivariate changes (Thorand et al., 2006). The increase in sE-selectin concentrations found during our study in patients with well-controlled type 2 diabetes and with uncontrolled diabetes proves endothelial activation, which seems to be related to the inflammation on the surface of the endothelium.

In the present study, STM levels were higher in patients with uncontrolled diabetes compared with the patients with well-controlled diabetes. A review of the literature suggests that the plasma concentration of sTM considerably increased during diabetes types 1 and 2 and in patients with extensive damage of the vascular endothelium in atherosclerosis, ischemic heart disease, disseminated intravascular coagulation, chronic renal failure, liver diseases, malaria, sepsis, and multiple organ injury or Graves' disease (Nadar et al., 2004). 
Marcos et al. (2001), studying a group of 49 patients with diabetes (34 with type 1 and 15 with type 2), observed an increase in plasma level of sTM during an 18-month follow-up. Furthermore, a significant increase in the concentration of albumin in the urine was reported in this group, again after 18 months of follow-up, which could indicate damage to the glomerular structure under the influence of hyperglycemia-induced oxidative stress, and this is considered the primary factor leading to the development of diabetic nephropathy. In the present study, increased levels of sTM were observed in patients with uncontrolled glycemia with micro-albuminuria, showing that hyperglycemia, ongoing inflammation, and oxidative stress may intensify the process of endothelial cell activation, which results in the disconnection of a fragment of supra-epithelial TM receptor and an increase in the soluble fraction of TM in the blood.

\section{Conclusions}

The study confirmed the participation of the inflammatory process associated with impaired vascular endothelial function in the pathogenesis of type 2 diabetes. The increase in sE-selectin concentrations found in our study in patients with well-controlled type 2 diabetes and with uncontrolled diabetes proves endothelial activation, which seems to be related to inflammation on the surface of the endothelium. Moreover, the opposite effect of uncontrolled hyperglycemia on adhesion molecules suggests different functions of VCAM-1 and ICAM-1 in complications of diabetes.

\section{Compliance with ethics guidelines}

Barbara RUSZKOWSKA-CIASTEK, Alina SOKUP, Tomasz WERNIK, Zofia RUPRECHT, Barbara GÓRALCZYK, Krzysztof GÓRALCZYK, Grażyna GADOMSKA, and Danuta ROŚĆ declare that they have no conflict of interest.

All procedures followed were in accordance with the ethical standards of the responsible committee on human experimentation (institutional and national) and with the Helsinki Declaration of 1975, as revised in 2008 (5). Informed consent was obtained from all patients for being included in the study. Additional informed consent was obtained from all patients for whom identifying information is included in this article.

\section{References}

Booth, G., Stalker, T.J., Lefer, A.M., et al., 2002. Mechanisms of amelioration of glucose-induced endothelial dysfunction following inhibition of protein kinase $\mathrm{C}$ in vivo. $\mathrm{Di}$ abetes, 51(5):1556-1565. [doi:10.2337/diabetes.51.5.1556]

Bruno, C.M., Valenti, M., Bertino, G., et al., 2008. Plasma ICAM-1 and VCAM-1 levels in type 2 diabetic patients with and without microalbuminuria. Minerva. Med., 99(1):1-5.

Empen, K., Frost, R.J., Geiss, H.C., et al., 2003. Differential effect of fenofibrate versus atorvastatin of E-selectin and vascular cellular adhesionmolecule-1 in patients with type 2 diabetes mellitus and mixed hyperlipoproteinemia: a randomized cross-over trial. Cardiovasc. Diabetol., 2:17. [doi:10.1186/1475-2840-2-17]

Głowińska, I., Małyszko, J., 2008. Cardiovascular complications in patients with diabetic nephropathy. Przegl. Kardiodiab., 3(3):217-222 (in Polish).

Głowińska-Olszewska, B., Urban, M., Peczyńska, J., et al., 2007. Analysis of selected new biomarkers of early atherosclerosis process in children and adolescents with diabetes type 1. Przegl. Kardiodiab., 2:146-153 (in Polish).

Grande, M.T., Pérez-Barriocanal, F., López-Novoa, J.M., 2010. Role of inflammation in túbulo-interstitial damage associated to obstructive nephropathy. J. Inflamm., 7(1):19. [doi:10.1186/1476-9255-7-19]

Güler, S., Cakir, B., Demirbas, B., et al., 2002. Plasma soluble intercellular adhesion molecule 1 levels are increased in type 2 diabetic patients with nephropathy. Horm. Res., 58(2):67-70. [doi:10.1159/000064664]

Hadi, A.R., Suwaidi, J., 2007. Endothelial dysfunction in diabetes mellitus. Vasc. Health Risk Manag., 3(6):853876.

Karalliedde, J., Gnudi, L., 2011. Endothelial factors and diabetic nephropathy. Diabetes Care, 34(Suppl. 2):S291S296. [doi:10.2337/dc11-s241]

Lu, J., Randell, E., Hun, Y., et al., 2011. Increased plasma methylglyoxal level, inflammation, and vascular endothelial dysfunction in diabetic nephropathy. Clin. Bioch., 44(4):307-311. [doi:10.1016/j.clinbiochem.2010.11.004]

Luis-Rodríguez, D., Martínez-Castelao, A., Górriz, J.L., et al., 2012. Pathophysiological role and therapeutic implications of inflammation in diabetic nephropathy. World $J$. Diabetes, 3(1):7-18. [doi:10.4239/wjd.v3.i1.7]

Mizia-Stec, K., Gąsior, Z., Zahorska-Markiewicz, B., et al., 2003. Immune activation in diabetes type 2 in patients with coronary artery disease. Diabetologia Doświadczalna i Kliniczna, 3(6):523-529 (in Polish).

Morcos, M., Borcea, V., Isermann, B., et al., 2001. Effect of $\alpha$-lipoic acid on the progression of endothelial cell damage and albuminuria in patients with diabetes mellitus: an exploratory study. Diabetes Res. Clin. Pract., 52(3):175183. [doi:10.1016/S0168-8227(01)00223-6]

Nadar, S., Blann, A.D., Lip, G.Y., 2004. Endothelial dysfunction: 
methods of assessment and application to hypertension. Curr. Pharm. Des., 10(29):3591-3605. [doi:10.2174/138 1612043382765]

Pupek-Musialik, D., Bogdański, P., Dytfeld, J., et al., 2005. Assesment of tumor necrosis factor soluble receptor type 2 (sTNFR2) and soluble intercellular adhesion molecule 1 (sICAM-1) in obese patients with metabolic syndrome. Inflammatory process in metabolic syndrome. Endokrynol. Otyt. Zaburz. Przem. Materii, 1(1):30-35 (in Polish).

Salker, T.J., Skvarka, C.B., Scalia, R., 2003. A novel role for calpains in the endothelial dysfunction of hyperglycemia. FASEB J., 17(11):1511-1513. [doi:10.1096/fj.02-1213fje]

Stehouwer, C.D., Gall, M.A., Twisk, J.W., et al., 2002. Increased urinary albumin excretion, endothelial dysfunction, and chronic low-grade inflammation in type 2 diabetes: progressive, interrelated, and independently associated with risk of death. Diabetes, 51(4):1157-1165. [doi:10.2337/diabetes.51.4.1157]

Szmit, S., Opolski, G., 2006. Diabetic microangiopathy-a current look at the pathogenesis and the clinical implications for cardiovascular diseases. Przegl. Kardiodiab., 1(1):27-34 (in Polish).

Thorand, B., Baumert, J., Chambless, L., et al., 2006. Elevated markers of endothelial dysfunction predict type 2 diabetes mellitus in middle-aged men and women from the general population. Arterioscler. Thromb. Vasc. Biol., 26(2): 398-405. [doi:10.1161/01.ATV.0000198392.05307.aa]

Żukowska-Szczechowska, E., Wystrychowski, G., 2007. Diabetic nephropathy and cardiovascular risk. Przegl. Kardiodiab., 2(4):209-213 (in Polish).

\section{中文概要}

题 目: 未受控制的高血糖对 2 型糖尿病患者粘附分子的 影响

目 的：评估可溶性血管细胞间黏附分子 (sVCAM-1)、 可溶性细胞间黏附分子 (sICAM-1) 、可溶性选 择素 $\mathrm{E}$ 和可溶性血栓调节蛋白在血糖控制良好和 不受控制的 2 型糖尿病患者中的水平。

创新点: 对 2 型糖尿病患者的血管内皮炎症标记物进行评 估。

方 法: 62 例糖尿病患者分成两组: 第一组包括 35 个血 糖控制良好的糖尿病患者, 第二组包括 27 个未 控制血糖并伴有微蛋白尿的糖尿病患者。对照组 由 25 名健康志愿者组成。测定血浆中 sVCAM- 1 、 sICAM-1、可溶性选择素 E 和可溶性血栓调节蛋 白的浓度, 同时测定血清肌䣶及血浆中空腹血糖 和糖化血红蛋白 (HbAlc) 的浓度。

结 论: 与血糖控制良好的糖尿病组相比, 未控制血糖组 具有相对低的 ICAM- 1 水平和更高的 VCAM- 1 水 平。未控制血糖组中患者的糖化血红蛋白和 ICAM-1 之间呈正相关, 肾小球滤过率和可溶性 选择素 E 之间呈正相关, 而肌䣶和 ICAM-1 之间 呈负相关。研究证实 2 型糖尿病的发病机理中炎 症过程的出现与血管内皮功能受损有关。未受控 制的高血糖对粘附分子的反向作用表明, 在糖尿 病的并发症中 VCAM-1 和 ICAM-1 具有不同功能。

关键词: 糖尿病肾病; 肾小球滤过率; 高滤过 